\title{
Challenge to establish the liver transplantation in developing country (Mongolia) progress of the liver transplantation program in Mongolia
}

\author{
Undarmaa Zandanbazar, Batsaikhan Bat-Erdene, Bat-Ireeduu Badarch, Batsaikhan Batsuuri, Sergelen Orgoi
}

Department of Organ transplantation, First Central Hospital of Mongolia, Ulaanbaatar, Mongolia

Background: The history of organ transplantation in Mongolia dates back to 10 years. The number of patients with end stage liver disease increased in worldwide and Mongolia has high prevalence of hepatitis B virus, hepatitis C virus and hepatocellular carcinoma. The most effective treatment in end stage liver disease is liver transplantation (LT). It has a lot of difficulties including high financial cost, lifelong follow-up treatment and affordable choice of health insurance system in inception and setting up of LT project in developing country such as Mongolia. In Mongolia, LT project has been started from 2011 and continued successfully.

Methods: Totally 106 patients had transplanted liver from living donor, and one patient from deceased donor. Cooperation of LT project between Mongolian National University of Medical Science, Mongolian First Central Hospital and Asan Medical Center, nowadays project is going successfully.

Results: Twenty-eight of the 99 cases of LT have performed by mixed LT team, and seven other cases performed by Mongolian $\mathrm{LT}$ team. Thirty-four patients were received from living donor $\mathrm{LT}$, and one was received from deceased donor. All of the patients who transplanted from living donor were survived and three patients died due to the laboratory deficits and, inadequate knowledge of patients. The complications which we faced were acute rejection, infection, biliary complication and sinusoidal obstructive syndrome.

Conclusions: Government support, affordable choice of health insurance system and demonstrating by experienced LT team were an important for evolving LT in low-income or developing country such as Mongolia. 